\title{
An Indoor Localization Algorithm Based on Convex Optimization under NLOS Environment
}

\author{
Long Zhang ${ }^{1 *}$,Pinpin $\mathrm{Lv}^{1}$,Chunqi Jiang ${ }^{1}$ \\ 1. China National Digital Switching System Engineering and Technological Research Center \\ Zhengzhou, China \\ 1160941757@qq.com,15903676771@163.com,pinghuyuephy@163.com
}

\begin{abstract}
In the actual indoor localization system, the existence of non-line-of-sight (NLOS) errors is a very important reason for the reduction of localization accuracy Therefore, how to deal with NLOS error becomes a research hotspot in the study of localization technology. In this paper, a robust quadratically constrained quadratic programming (QCQP) method is proposed for NLOS error. The algorithm does not need to know the distribution information of NLOS errors, nor does it need to judge the NLOS status of each path, and the complexity is low, which has high practical application value.
\end{abstract}

\section{Keywords-NLOS; localization; robust; QCQP}

\section{INTRODUCTION}

With the increasing popularity of the location service market, as an important branch, indoor location services has gradually drawn the attention of many scholars and enterprises. A typical indoor positioning application can be divided into three parts. Firstly, obtaining the distance information between the sensor node A Indoor Localization Algorithm Based on Convex Optimization under NLOS Environment and the target node. Secondly, calculating the target position by the localization algorithm according to the distance information and the position of the known nodes. Finally, visualizing the targets combined with the map information.

For indoor localization technology, raising the ranging accuracy between sensor nodes and target nodes is an effective method to improve the localization accuracy. However, in the actual positioning system, the accuracy of indoor ranging will be affected by such factors as indoor multipath, non-line-ofsight (NLOS), hardware clock synchronization error and so on. In order to obtain more accurate localization results, we need to suppress and process the errors in the above ranging information, especially the NLOS errors. In this paper, we will mainly discuss the location algorithm in the NLOS environment. The commonly used methods for NLOS error suppression include maximum likelihood algorithm (ML) [1], least squares algorithm (LS), residual weighting algorithm (RWGH) [2] and so on. However, these methods need the prior information of NLOS error, such as NLOS error distribution, the NLOS status of each path. Because of the time-varying environment and the existence of NLOS status determination error, it is difficult to satisfy the above conditions in practical systems.

In this paper, we focus on finding a localization algorithm that can effectively suppress NLOS errors without obtaining NLOS information. Considering the practicability and localization accuracy of localization algorithm, we decided to design a localization algorithm based on convex optimization Wang et al. proposed three convex optimization methods using second-order cone relaxation, semi-positive definite relaxation and robust semi-positive definite relaxation. These methods can still suppress the effect of NLOS error on localization without NLOS information, but there are some robustness problems which will not find the localization solution with a certain probability, and the algorithm is of high complexity. Aiming at the problems of the above methods, this paper proposes a localization algorithm based on second-order quadratic programming which is robust, accurate and practical. It can well meet the needs of practical indoor localization applications.

\section{PROBLEM FORMULATION}

\section{A. The Model of Localization Algorithm}

In order to meet the actual localization needs, we need three sensor nodes at least, so this paper assumes that there are $\mathrm{N}$ sensor nodes $(\mathrm{N}>=3)$, and a target node in indoor scenarios, and in this environment there is a situation of immediate occlusion, namely, NLOS situations between certain sensor nodes and the target node. Assuming that the true coordinate of the target node is $\mathbf{x}$, the coordinates of sensor nodes are $\mathbf{x}_{i}$, the measurement distance, measurement error and NLOS error of the target node and each sensor node are $d_{i}, n_{i}$ and $e_{i}$, respectively. For the convenience, we define the link between target node and $i$ th sensor node is the $i$ th link ( $i=1, \mathrm{~L}, N)$.We can see that the following relations exist.

$$
d_{i}=\left\|\mathbf{x}-\mathbf{x}_{i}\right\|+n_{i}+e_{i}, \quad i=1, \ldots, N
$$

To facilitate the analysis of the problem, we put forward the following assumptions:

(1) $n_{i}: N\left(0, \sigma_{i}^{2}\right)$. That is, the measurement error of each link follows a zero-mean Gaussian distribution;

(2) The NLOS errors are invariably non-negative, and there are $N_{n}$ NLOS links in all $N$ links. When the link is line-of-sight (LOS), we have $e_{i}=0$, and given the upper bound of NLOS error, i.e. $\max \left(e_{i}\right) \leq \rho$;

It is noteworthy that we find most algorithms via convex optimization often use the following assumption (a)[3][4][6]: the measurement noise $n_{i}$ much less than NLOS error $e_{i}$. 
However, there are some defects in this assumption, which will lead to the unsolvable problem in the localization. We will further explain this problem and improve it in the following sections.

\section{B. Derivation of Convex Optimization Model}

From the (1.1), we can get the following deformation.

$$
n_{i}=d_{i}-e_{i}-\left\|\mathbf{x}-\mathbf{x}_{i}\right\|
$$

Hence, we can transform the node position estimation into a typical LS problem:

$$
\min _{x} \sum_{i=1}^{N} \frac{\left(d_{i}-e_{i}-\left\|\mathbf{x}-\mathbf{x}_{i}\right\|\right)^{2}}{\sigma_{i}^{2}}
$$

In order to get a more robust localization algorithm, we transform it into a RLS problem.

$$
\min _{x} \max _{\left\{e_{i}\right\}} \sum_{i=1}^{N} \frac{\left(d_{i}-e_{i}-\left\|\mathbf{x}-\mathbf{x}_{i}\right\|\right)^{2}}{\sigma_{i}^{2}}
$$

Define a function: $g\left(e_{i}\right)=\left|d_{i}-e_{i}-\left\|\mathbf{x}-\mathbf{x}_{i}\right\|\right|$. So, we have

$$
\min _{x} \max _{\left\{e_{i}\right\}} \sum_{i=1}^{N} \frac{g^{2}\left(e_{i}\right)}{\sigma_{i}^{2}} .
$$

Clearly known

$$
\max _{\left\{e_{i}\right\}} \sum_{i=1}^{N} \frac{g^{2}\left(e_{i}\right)}{\sigma_{i}^{2}}=\sum_{i=1}^{N} \frac{\left(\max _{e_{i}} g\left(e_{i}\right)\right)^{2}}{\sigma_{i}^{2}}
$$

Because of $0 \leq e_{i} \leq \rho,(6)$ can be rewrite as

$$
\max _{e_{i}} g\left(e_{i}\right)=\max \{g(0), g(\rho)\}
$$

Combined (7), the question (5) is reformulated as:

$$
\begin{aligned}
& \min _{x} \sum_{i=1}^{N} \frac{\lambda_{i}^{2}}{\sigma_{i}^{2}} \\
& \text { s.t. } g(0)=\left|d_{i}-\left\|\mathbf{x}-\mathbf{x}_{i}\right\|\right| \leq \lambda_{i}, i=1, \mathrm{~K}, N, \\
& \quad g(\rho)=\left|d_{i}-\| \mathbf{x}-\mathbf{x}_{i}\right||-\rho| \leq \lambda_{i}, i=1, \mathrm{~K}, N
\end{aligned}
$$

Constraints (9), (10) can be further expressed as

$$
d_{i}-\lambda_{i} \leq\left\|\mathbf{x}-\mathbf{x}_{i}\right\| \leq d_{i}+\lambda_{i}-\rho, i=1, \mathrm{~K}, N
$$

The assumption (a) mentioned above is usually used as the following constraint:

$$
\left\|\mathbf{x}-\mathbf{x}_{i}\right\| \leq d_{i}
$$

This constraint needs to satisfy $e_{i}+n_{i}$ is non negativity. However, we can not make this judgment due to the randomness and uncertainty of NLOS errors in the actual localization system. Therefore, this paper adopts another constraint method with high robustness.

$$
\left\|\mathbf{x}-\mathbf{x}_{i}\right\| \leq d_{i}+\mu_{i}
$$

We know that a random variable with normal distribution has a probability of more than $95 \%$ distributed in the range of two standard deviation of the mean value. Therefore, according to assumption (1) that the measurement error obeys the normal distribution, we can get:

$$
P\left(\left\|\mathbf{x}-\mathbf{x}_{i}\right\| \leq d_{i}+\mu_{i} \mid \mu_{i}=2 \sigma_{i}\right) \approx 0.955
$$

Combining (12) with (13), we get the new constraint via as follows:

$$
A[\mathbf{x} ; r] \leq f
$$

Where

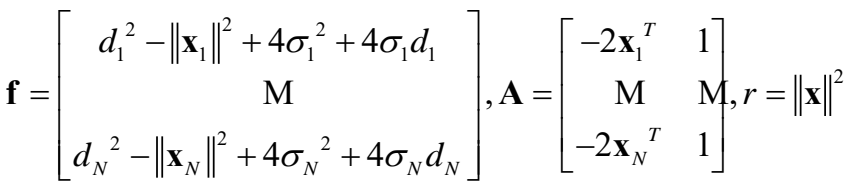

At this point, we can get the following optimization problems:

$$
\begin{array}{ll}
\min _{\mathbf{x}, r,\left\{\lambda_{i}\right\}} & \sum_{i=1}^{N} \frac{\lambda_{i}^{2}}{\sigma_{i}^{2}} \\
\text { s.t. } & d_{i}-\lambda_{i} \leq\left\|\mathbf{x}-\mathbf{x}_{i}\right\|, \\
& \left\|\mathbf{x}-\mathbf{x}_{i}\right\| \leq d_{i}+\lambda_{i}-\rho, \\
& A[\mathbf{x} ; r] \leq f, \\
& r=\|\mathbf{x}\|^{2}, i=1, \mathrm{~K}, N
\end{array}
$$

Because the equality constraint is not affine and the second inequality constraint is concave, problem (17) is still nonconvex and difficult to solve by using correlation convex optimization tools. Therefore, we replace $r=\|\mathbf{x}\|^{2}$ with $r \geq\|\mathbf{x}\|^{2}$ and introduce the new variable $B_{i}$ to obtain convex optimization problems as follows:

$$
\begin{array}{ll}
\min _{\mathbf{x}, r,\left\{\lambda_{i}\right\}, B_{i}} & \sum_{i=1}^{N} \frac{\lambda_{i}^{2}}{\sigma_{i}^{2}} \\
\text { s.t. } & \lambda_{i}^{2}-2 d_{i} \lambda_{i}-r+2 \mathbf{x}^{T} \mathbf{x}_{i} \leq\left\|\mathbf{x}_{i}\right\|^{2}-d_{i}^{2}, \\
& r-2 \mathbf{x}^{T} \mathbf{x}_{i}-A_{i} \leq-\left\|\mathbf{x}_{i}\right\|^{2}, \\
& \left(d_{i}+\lambda_{i}-\rho\right)^{2} \leq A_{i}, \\
& A[\mathbf{x} ; r] \leq f \\
& r \leq\|\mathbf{x}\|^{2}, i=1, \mathrm{~K}, N
\end{array}
$$

\section{Simulation Results}

In order to better analyze the algorithm in this paper, we choose three representative convex optimization algorithms as comparison algorithms which are semidefinite programming, robust semidefinite programming [4] and robust second-order cone programming [6]. In the following sections, we represent these algorithms as "SDP", "SDP Robust" and "SOCP-Robust", respectively. And the algorithm we proposed is represented by "QCQP". Assume that all sensor nodes are distributed in the 
area of $10 \times 10 \mathrm{~m}^{2}$. In this area, there are randomly distributed $N=5$ positioning nodes and 1 target nodes. $M=3000$ Monte Carlo experiments are carried out for the location results under each condition. For convenience of analysis, we also assume that the measurement errors in each environment obey the same Gauss distribution, namely, $\sigma_{1}^{2}={\sigma_{2}}^{2}=\mathrm{L}=\sigma_{N}{ }^{2}=\sigma^{2}$. Consulting the simulation condition in [6], we consider that all NLOS errors obey a uniform distribution, that is, $e_{i}: \mu(0, \rho)$.

The localization algorithm performance evaluation mainly uses two indicators: the localization accuracy which is expressed by root mean square error (RMSE) and the number of unsolved situation (NUS). All simulations run under the same conditions, which use the CVX toolbox of MATLAB, the solver is sedumi and the precision is best[5]. In this following, we will compare the two indicators of the above algorithm in two scenarios.

(1) Scenario 1: In this scenario, LOS links occupy the majority of all links. We assume that the count of NLOS links is 1 , namely, $N_{n}=1$, and the rest is LOS, the upper bound of NLOS errors $\rho$ is $5 \mathrm{~m}$. Varying the measurement noise standard deviation (STD) from 0.15 to 0.9 , Fig. 1 shows the localization accuracy of the four convex algorithms.

The NUS of each algorithm under different STD is shown in TABLE I. Because the constraints in the first three algorithm contain the deformation of assumption (a) mentioned above, the reliability of the constraint becomes weaker when LOS links is dominant, and it is easy to cause the situation that problem can not be solved. This algorithm does not use (a) as a constraint condition, but chooses to use constraints (15) which is more reliable, so it has better robustness.

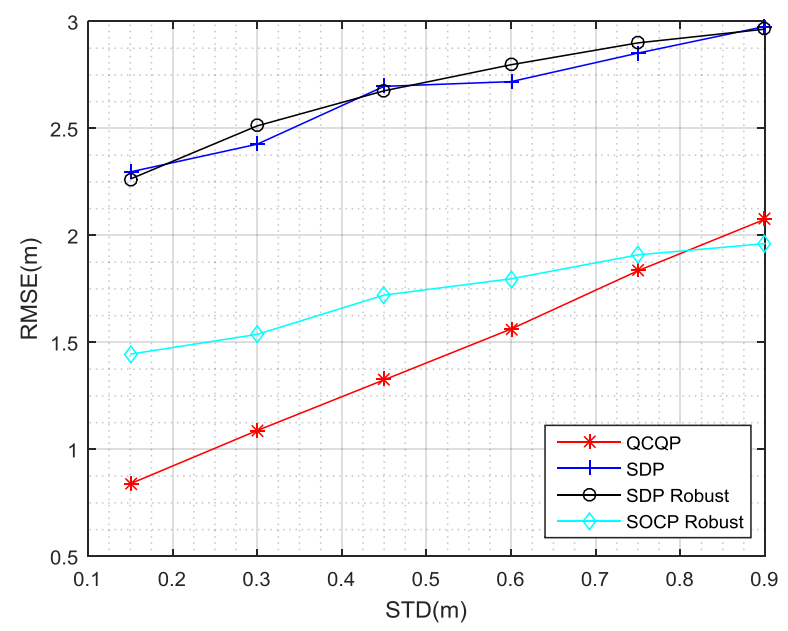

Fig.1 When $N_{n}=1$, the RMSE of four algorithms

(2) Scenario 2: In this scenario, NLOS links play a leading role. We set $N_{n}=4, \rho=5 m$. Fig. 2 shows the localization result with the STD changing from 0.15 to 0.9 . When the STD is small, we see that the performance of the convex optimization algorithm proposed in this paper is better than SDP and the SDP SDP-Robust, but with the increase of the STD, its performance gradually decreases. The performance of SOCP-Robust algorithm proposed in document SS is better than the above algorithms. This is because the assumption (a) in SOCP-Robust become more reliable as a result of the NLOS links increasing in the location. For this reason, we can also see that the NUS of SOCP-Robust in Scenario 2 is much smaller comparing with Scenario 1 from TABLE II. My convex is less accurate than SOCP-Robust due to the introduction of a new variable $B_{i}$. Table 3 shows that all the four algorithms have good robustness, especially my convex.

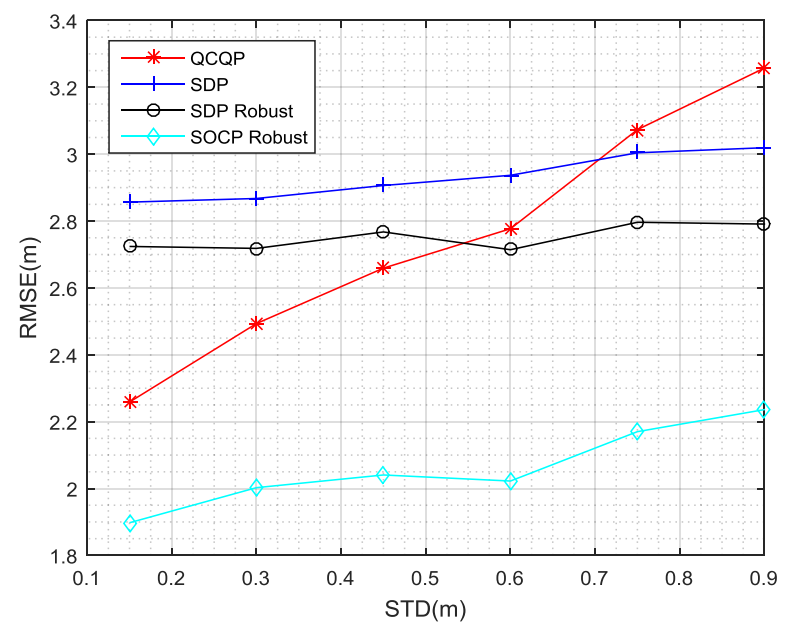

Fig.2. When $N_{n}=4$, the RMSE of four algorithms

TABLE I when $N_{n}=1$, the NUS of four algorithms

\begin{tabular}{|c|c|c|c|c|c|c|}
\hline Method & 0.15 & 0.3 & 0.45 & 0.6 & 0.75 & 0.9 \\
\hline SDP & 567 & 726 & 886 & 904 & 989 & 1031 \\
\hline SDP-Robust & 301 & 607 & 786 & 842 & 929 & 970 \\
\hline SOCP-Robust & 474 & 673 & 854 & 885 & 971 & 1016 \\
\hline QCQP & 0 & 1 & 7 & 4 & 6 & 5 \\
\hline
\end{tabular}

TABLE II when $N_{n}=4$, the NUS of four algorithms

\begin{tabular}{|c|c|c|c|c|c|c|}
\hline Method & 0.15 & 0.3 & 0.45 & 0.6 & 0.75 & 0.9 \\
\hline SDP & 15 & 35 & 66 & 103 & 134 & 207 \\
\hline SDP Robust & 7 & 27 & 57 & 94 & 117 & 184 \\
\hline SOCP-Robust & 13 & 32 & 68 & 104 & 129 & 207 \\
\hline QCQP & 0 & 0 & 0 & 0 & 0 & 0 \\
\hline
\end{tabular}

(3) Scenario 3: Although the proposed algorithm has strong robustness advantages, its localization accuracy is still worse than SOCP-Robust with the increase of NLOS links in localization scenario. While SOCP-Robust is relatively small in NLOS connection, the NUS increases sharply, which is 
difficult to meet the actual location requirements. So we replacing the constraint (13) of problem (15) in [6] with (15) in this paper, a new convex algorithm, "SOCP-Re", is proposed based on SOCP-Robust. The localization performance of SOCP-Re is simulated as follows.

Setting simulation conditions such as Scenario 1 and Scenario 2, we can get the comparison of SOCP-Robust and SOCP-Re localization accuracy as shown in Fig.3 and Fig.4 respectively. From Fig.3 and Fig.4, we can see that SOCP Re localization accuracy is not as good as SOCP-Robustness due to looser constraints (15), but SOCP-Re has good performance. In both scenarios, The NUS performance of SOCP-Re is shown in TABLE III under scenario 1 and scenario 2, and we can see that SOCP-Re performs better than SOCP-Robust. SOCP-Re algorithm can achieve a compromise between positioning accuracy and robustness due to the introduction of new constraints, which can better meet the needs of the actual localization system.

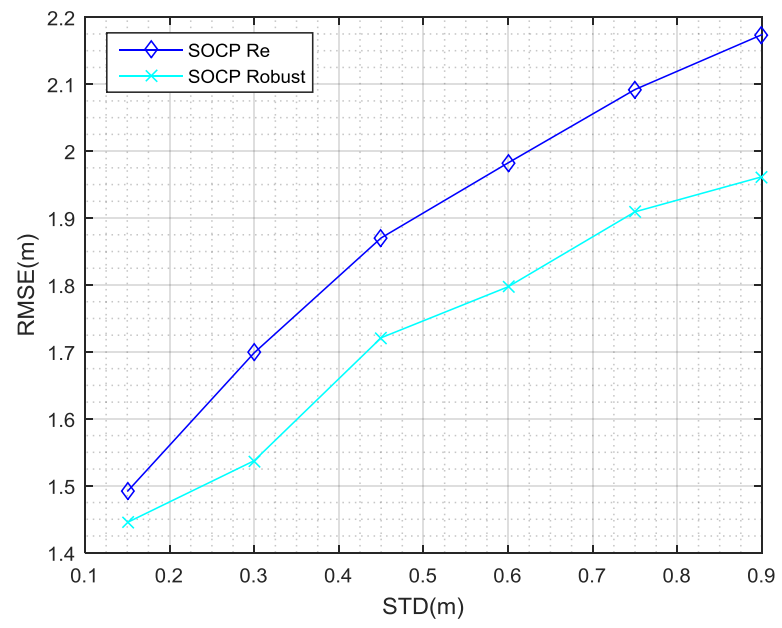

Fig.3 The RMSE between SOCP-Re and SOCP-Robust under Scenario 1

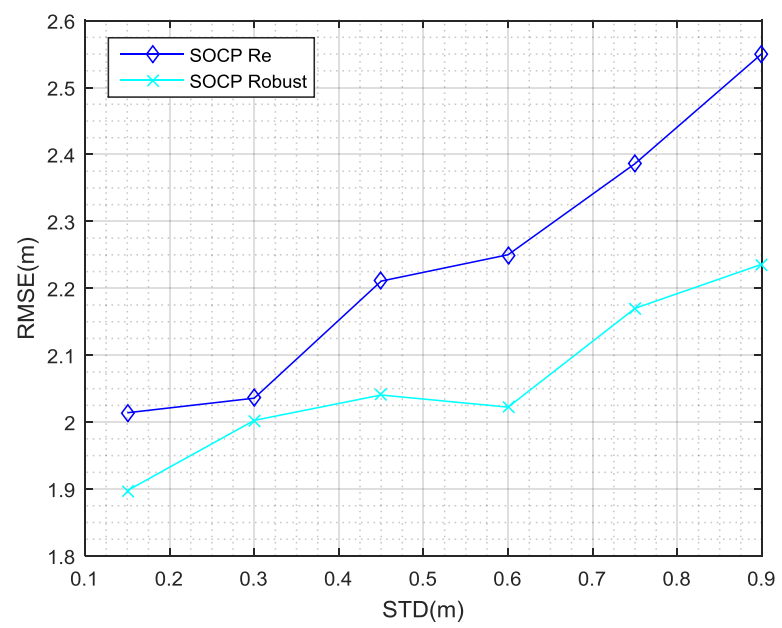

Fig.4 The RMSE between SOCP-Re and SOCP-Robust under Scenario 2
TABLE III. The NUS between SOCP-Re and SOCP-Robust under different scenarios

\begin{tabular}{|c|c|c|c|c|c|c|}
\hline$\sigma$ & 0.15 & 0.3 & 0.45 & 0.6 & 0.75 & 0.9 \\
\hline Scenario 1 & 0 & 2 & 2 & 4 & 4 & 3 \\
\hline Scenario 2 & 0 & 0 & 0 & 0 & 0 & 0 \\
\hline
\end{tabular}

\section{CONCLUSIONS}

Aiming at the NLOS error in indoor localization, a robust QCQP positioning method is introduced in this paper. This method does not need to know the specific NLOS error distribution information, nor does need to identify the NLOS links. The robustness and accuracy of the QCQP are further verified in the simulation test. In addition, this paper improves on the basis of SOCR robustness and obtains a more robust and practical SOCR-Re algorithm at the cost of certain localization accuracy.

\section{REFERENCES}

[1] S. Gezici and Z. Sahinoglu, "UWB geolocation techniques for IEEE 802.15.4a personal area Networks," Mitsubishi Electric Research Laboratories (MERL) Tech. Rep., Cambridge, MA, USA, Aug. 2004.

[2] P. C. Chen, "A non-line-of-sight error mitigation algorithm in location estimation," in Proc. IEEE Int. Conf. WCNC, New Orleans, LA, USA, Sep. 1999, vol. 1, pp. 316-320

[3] S. Gao, F. Zhang and G. Wang, "NLOS Error Mitigation for TOABased Source Localization With Unknown Transmission Time," in IEEE Sensors Journal, vol. 17, no. 12, pp. 3605-3606, 15 June15, 2017.

[4] G. Wang, H. Chen, Y. Li and N. Ansari, "NLOS Error Mitigation for TOA-Based Localization via Convex Relaxation," in IEEE Transactions on Wireless Communications, vol. 13, no. 8, pp. 4119-4131, Aug. 2014.

[5] M. Grant and S. Boyd, CVX: Matlab Software for Disciplined Convex Programming. [Online]. Available: http://stanford.edu/ boyd/cvx.

[6] S. Zhang, S. Gao, G. Wang and Y. Li, "Robust NLOS Error Mitigation Method for TOA-Based Localization via Second-Order Cone Relaxation," in IEEE Communications Letters, vol. 19, no. 12, pp. 2210 2213, Dec. 2015. 